Anm. Zootech., 1961, 10 (3), 2I5-227.

\title{
OBSERVATIONS SUR LES QUALITÉS ORGANOLEPTIQUES DE POULETS APPARTENANT A UNE MÊME SOUCHE
}

\author{
F. II. RI(ARI) \\ Station de Recherches anicoles, Domaine expérimental du .Magneraud, \\ St-Pierre d'Amilly (C'harente-Maritime).
}

SOMMAIRE

Lés qualités organolept iques de go coquelets appartenant à la mème souche ont été étudiées à l'aide des tests de dégustation. Le jury était composé de 4 hommes et + femmes choisis parmi 34 volontaires, d'après leur aptitude à déceler le goût de poulet dans un jus dilué et la fidélité de leur jugement, apprécié au cours de 2 dégustations d'entraînement. Less séances portaient chacune sur 4 poulets. Pour chacun d'eux, les dégustateurs devaient juger un morceau de blanc, un morceau de cuisse et un morceau de peau. Ils en notaient la saveur, l'intensité du goût, la tendreté, la jutosité et la texture. à l'aide d'échelles clescriptives. Les 4 poulets étaient ensuite classés par ordre de préférence puur chacun des 3 morceatux.

Le's résultats montrent que les caractéristiques de la peau sont plus variables que celles de la cuisse et du blanc. Dans chaque cas, la saveur présente le plus faible coefficient de variation. La valeur relative des morceaux dépend du caractère envisagé, mais, dans l'ensemble, la cuisse est le morceaul le mieux apprécié. J.es corrélations phénotypiques entre morceaux sont légèrement positives ou non significatives. Entre caractères organoleptígues elles sunt en général assez fortes. Enfin, la méthode utilisée n’a pas permis de mettre en évidence une variabilité génétique bien nette et aucune valeur calculée pour l'héritabilité n'est significative.

\section{INTRODUC'TION}

Parmi les nombreux aspects de la qualité du poulet, le plus important est sans conteste celui concernant les caractères organoleptiques. Depuis fort longtemps, de nombreux aviculteurs français ont cherché à améliorer cette qualité, d'une façon fort empirique, ce qui a abouti à de véritables " crus " dont le plus connu est sans doute le poulet de Bresse.

Mais le déterminisme de la qualité organoleptique reste très mal connu. D'une part c'est une question très complexe. Ainsi, on connaît mal les substances responsables du goît de poulet (Pippen, Campbeil, et StrReeter, 1954). De plus, de profondes modifications histologiques et biochimiques apparaissent après le sacrifice et influent sur les caractéristiques de qualité (LOWE, I948; I) E FrEMERY et PoOL, r960). D'autre part la méthode directe de mesure, qui consiste à faire juger les

Aunales de Zootechnie. - ig6r. 
animaux par un jury de dégustateurs est une méthode peu précise, longue et délicate à mettre en œuvre. MARSDEN et al. (I952) estiment même que les observations des dégustateurs sont trop peu précises pour qu'on puisse leur appliquer les calculs statistiques.

Jusqu'à présent, on a surtout étudié l'action sur la qualité organoleptique de facteurs tels que l'alimentation, les techniques d'abattage ou de conditionnement. Peu de travaux concernent la variabilité entre souches, entre familles ou entre individus d'une même souche, alors que c'est la base de toute amélioration génétique. De plus, ces travaux conduisent souvent à des résultats peu probants. Ainsi la comparaison des poulets et des régimes utilisés en Amérique respectivement en I930 et en 1956 n'a permis de mettre en évidence des différences significatives ni pour la saveur (HANSON et al., I959) ni pour la tendreté (GILPIN et al., I960).

Pour essayer d'approfondir ce problème, nous avons analysé les observations données par un jury de 8 dégustateurs à propos de 90 coquelets pris parmi 30 familles d'une même souche expérimentale, puis nous avons comparé nos résultats avec ceux d'autres auteurs.

\section{MATÉRIEL ET MÉTHODES}

\section{I - Choix el préparation des animaux.}

Les go coquelets étaient issus, en reproduction pédigrée, de 10 coys et 30 poules, soient 3 poules par coq, 3 enfants analysés pour une même poule et 9 pour un même cọ. La souche utilisée était une population encore hétérogène, d'où, à priori, une variabilité génétique relativement importante.

Les 30 familles ont été choisies d'après le nombre total de poussins éclos et la régularité des éclosions, de façon à avoir sûrement plus de 3 fils à l'âge d'abattage. A l'intérieur des familles, les animaux ont été pris au hasard, ì deux restrictions près : les poulets les plus beaux étaient gardés en vue de la reproduction et n'ont pu être utilisés ; les poulets trop petits (moins de $600 \mathrm{~g}$. à $8 \mathrm{se}$ maines) ont été systématiquement écartés car ce sont en général des animaux malades, peu représentatifs de la valeur réelle de la souche.

Le prélèvement s'est fait dans les 8 lots, éclos du 22 juin au 10 août Ig6o, correspondant à la saison de reproduction normale de cette souche. Les lots 1 à 7 ont fourni chacun 12 poulets et le lot 8,6 . Tous les animaux ont été élevés dans une même poussinière. Ils ont subi les mêmes traitements et reçu la même alimentation (type basse énergie et sans antibiotique).

Les 90 poulets ont été sacrifiés au même âge ( 12 semaines et 3 jours), après environ 15 heures de jeûne complet. Ils ont été saignés par section des vaisseaux au fond de la gorge, échaudés pendant une minute environ dans de l'eau à $53^{\circ} \mathrm{C}$ et plumés avec une plumeuse à doigts de type artisanal, les restes de plumage étant enlevés à la main. Après avoir été effilés, on les a laissés refroidir pendant environ 8 heures à la température du laboratoire. On les a alors entreposés dans un réfrigérateur de type ménager à une température voisine de $+4^{\circ} \mathrm{C}$ jusqu'à leur utilisation, soit 2,3 ou 4 jours.

Avant la cuisson, tous les viscères étaient enlevés sauf les reins et les poumons. Les pattes étaient coupées au niveau de l'articulation tibia-tarsométatarse et le cou entre la onzième et la douzième vertèbre cervicale.

Le poulet était ensuite bridé et placé sur la broche d'une rotissoire électrique horizontale à air libre. La cuisson était arrêtée quand le poulet paraissait cuit à point.

Nous n'avons pas fait d'essais préliminaires pour voir si les procédés d'abattage, de conservation ou de cuisson utilisés avaient une influence favorable ou défavorable sur les qualités organoleptiques. Nous nous sommes seulement attaché à rendre aussi uniforme que possible l'action de ces divers facteurs sur tous les poulets.

\section{2 - Organisation des dégustations.}

Les séances ont eu lieu 3 fois par semaine (lundi, mardi et mercredi à I I heures) pendant 8 semaines consécutives, dans une salle différente de celle où avait lieu la cuisson. Chacune portait 
sur 4 poulets fils de 4 coqs différents. Les dégustateurs recevaient successivement un échantillon de peau des 4 poulets, un norceau des 4 blancs et un morceau des 4 cuisses. La peau était prélevée sur le dos de l'animal où elle se présente d'une facon homogène sur une assez grande surface ; le morceau de blanc était découpé dans le muscle Pectoralis superficialis; la viande de la cuisse était un ensemble des 3 muscles Biceps femoris, Semimembranosus et Semitendinosus (nomenclature de CHAMBERLAIN, I 943). Les morceaux étaient dégustés chauds, dans des assiettes légèrement réchauffées. Chaque échantillon était désigné par un numéro de code et l'ordre des poulets était différent pour chacune des 3 séries.

Après chaque morceau, les dégustateurs devaient manger une tranche de pomme afin d' " effacer "le goût du morceau précédent. Ils avaient aussi à leur disposition, mais de façon facultative, de l'eau et du pain. Cette opération, également pratiquée par BAKER et al. (1956) permet de mettre le palais du dégustateur à peu près dans les mêmes conditions pour tous les morceaux analysés au cours d'une séance.

Une correspondance morceau-dégustateur a été établie de façon que, pour un dégustateur domné, les morceaux aient sur la carcasse la même position relative pour les 90 poulets.

$$
3 \text { - La Nolation. }
$$

Les caractéristiques organoleptiques le plus souvent étudiées dans les mémoires traitant de la qualité du poulet sont la saveur, la tendreté, et la jutosité. Puis viennent l'arôme, la texture et quelquefois la couleur de la viande. Nous avons laissé de côté couleur et arôme. Par contre, nous avons demandé aux dégustateurs d'apprécier l'intensité de ce qu'on peut appeler " le goût de poulet ". L'expérience présente portait donc sur 5 caractéristiques qui peuvent se définir de la façon suivante:

Saveur : propriété générale pour un échantillon d'être bon ou mauvais.

Intensité du goủt : propriété pour le morceau dégusté d'être fade ou de présenter plus ou moins le goût que les gens ont l'habitude de prêter au bons poulets.

I. N.R.A. Feuille de dégustation

lagneraud

Nom :

Date :

Échantillon :

Heure :

Jugez ce morceau de poulet d'après le plaisir que vous avez à le manger. Mettez une croix en face du not qui vous paraît convenir le mieux, pour chacun des caractéres indiqués.

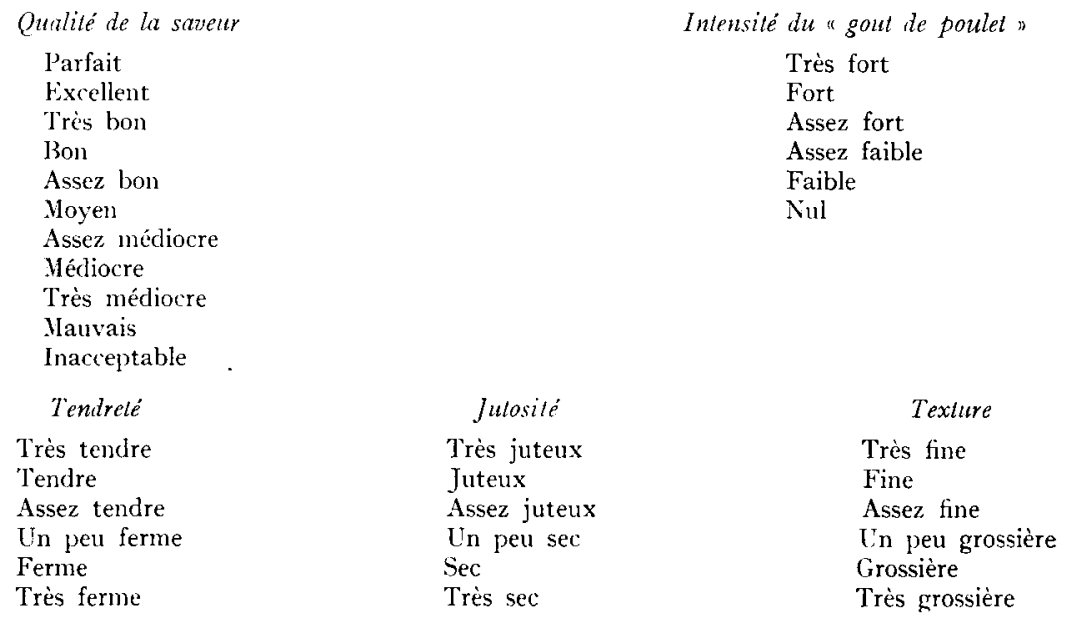

Observations:

FIs; r. - Fenille de note utilisée par un dégustateur pour un morceau. 
Tendrele' : propriété pour le morceau d'être plus ou moins résistant à la mastication.

Jutosite' : Propriété d'être juteux ou sec. Rend compte de la quantité de jus qui semble se sé. parer du morceau au cours de la mastication mais n'implique aucun jugenent simultané sur la (qualité de ce jus (dans ce dernjer cas, le terme "succulence " serait glus correct).

Texture : propriété liée à la finesse du "grain ", c'est-à-dire la structure fine ou grossière du morceau, provoquant au contact des murjueuses bucales une sensation physique agréable ou désagréable.

Pour matérialiser leur jugement, les dégustateurs disposaient de feuilles, une pour chaque morceau de chaque poulet, comprenant une échelle descriptive pour chacun des caractères à noter, sur lesquelles ils cochaient l'adjectif correspondant à leur appréciation (fig. I). Le problème des échelles descriptives a été discuté par PLANK (I948) : le choix des termes doit être tel que de l'un à l'autre il $y$ ait toujours une égale différence de sensation. Notre échelle des saveurs a été directement adaptée de 2 exemples que donne cet auteur (échelle de RHeiviold, i940, pour les fruits et légumes; échelle de RAMsiotron, 1947, pour la viande). Pour les autres caractéristiques, nous avons essayé de suivre des directives de P'LANK en utilisant un simple étalement des dualités : goût fort ou faible, morceau tendre ou ferme, juteux ou sec, à texture fine ou grossière.

P'our avoir un complément d'information, nous avons demandé aux dégustateurs de classer par ordre de préférence sur une feuille séparée les 4 morceaux de peau, les 4 blancs et les 4 cuisses.

I.es observations obtenues ont été transformées en notes chiffrées de la façon suivante :

$I^{\circ}$ pour la saveur, chaque adjectif a été affecté d'une note allant de zéro pour "inacceptable » à ro pour "parfait ", en passant par 5 pour "moven", systène en accord avec la critique de P'IANK (1948);

$2^{\circ}$ pour les autres caractéristiques, les notes allaient également de zéro pour le plus mauvais à so pour le meilleur, mais seuls les nombres pairs ont été utilisés ;

$3^{\circ}$ pour les rangs de classement, chacun a été transformé en note normale selon la table XX de Fisher et Yates (1948) à laquelle on a jouté 5 pour éviter la manipulation de nombres négatifs.

Enfin, pour chaque poulet on a calculé la moyenne des appréciations des 8 dégustateurs pour chacun des 5 caractères des 3 morceaux, la moyenne arithmétique de toutes les notes par morceau et la moyenne des notes de rang par morceau.

$$
4 \text { - Le choix des dégustateurs. }
$$

\section{a) Principes directeurs.}

L'appréciation subjective d'une caractéristique organoleptique est peu précise ct soumise aux variations psychologigues individuelles. Les expérimentateurs font donc appel au plus grand nombre de dégustateurs possible afin de limiter l'incidence d'une erreur de jugement et d'obtenir un résultat valable pour une large couche de la population consommatrice du produit étudié. D'un autre côté, le nombre de morceaux qu'on peut découper dans un blanc ou une cuisse de poulet est limité. Pour la présente expérience, nous avons fixé à 8 le nombre des dégustateurs, pris également dans les 2 sexes et si possible de diverses conditions sociales.

Au point de vue méthodologie, la sélection des dégustateurs est probablement le problème le plus délicat. Nous avons donc réalisé une expérimentation préliminaire en suivant les directives de Girardot, IERyam et Silapiro (1952), directives qui peuvent se résumer de la façon suivante :

Io Il faut faire les tests de sélection avec le même matériel qui doit être utilisé dans les tests expérimentaux futurs et il est important que les candidats soient animés de bonne volonté.

$2^{\circ}$ On peut effectuer une première sélection d'après l'aptitude à séparer deux échantillons peu différents, en utilisant un test de différence simple. On éliminera ainsi ceux qui manquent de sensibilité ou de mémoire des saveurs.

$3^{\circ}$ Il faut ensuite tester l'aptitude à reproduire un jugement de qualité en faisant juger, pourdes échantillons différents, plusieurs morceaux du même échantillon et en calculant le rapport :

\section{Variabilité entre échantillons}

Variabilité intra-échantillon

Il s'agit d'une analyse de variance simple et du calcul du F (SNEDEcoR, 1956). Ce deuxième test permet d'éliminer ceux qui manquent de fidélité dans leur jugement ou qui ne comprennent par le mécanisme des dégustations.

\section{b) Organisation matérielle.}

Pour constituer notre jury, nous avons d'abord demandé des volontaires dans tous les services du Domaine du Magneraud. 34 personnes ont fait acte de candidature, ce qui représentait environ 50 p. 100 du personnel. 
Dans un deuxième temps la sensibilité des candidats au goût de poulet a été étudiée à l'aide de tests triangles (Io à 15 répétitions selon les candidats) au cours desquels on présentait aux candidats 2 gobelets contenant de l'eau du robinet et un troisième du jus de poulet très dilué $(2,5 \mathrm{p}$. I 00$)$. Le jus a été obtenu en faisant cuire au bain-marie bouillant pendant i I heures les muscles de la cuisse et de la poitrine d'un poulet. La viande cuite a été ensuite laissée au réfrigérateur pendant 36 heures avec une quantité d'eau égale à une fois et demie le poids des muscles frais. Après filtration et dilution, on a obtenu une préparation sans aucune différence d'aspect ni de couleur avec l'eau.

I 2 personnes ayant donné moins de 45 p. Ioo de bonnes réponses ont été éliminées. Dans un troisième temps les 22 autres ont été invitées à participer à 2 dégustations d'entraînement organisées sur le même modèle que les dégustations expérimentales, à 2 exceptions près : la peau n'a pas été dégustée et l'intensité du goût n'était pas notée. A chacune des 2 séances, les candidats devaient noter 2 morceaux voisins du même blanc et 2 morceaux voisins de la même cuisse, ceci pour 2 poulets d'origine différente : un coquelet type Cornish x Sussex, âgé de 8 semaines, provenant d'un élevage privé ; un coquelet de notre propre élevage, âgé de I4 semaines, ayant reçu un aliment à basse énergie et sans antibiotique. Chaque candidat a donc dégusté 2 fois un blanc et une cuisse de 2 poulets âgés et de 2 poulets jeunes. Nous avons fait une analyse de variance sur les appréciations transformées en notes chiffrées, en considérant que nous avions 2 répétitions de 8 traitements différents et nous avons calculé :

$$
F=\frac{\text { Variabilité entre traitements }}{\text { Variabilité entre observations }} \text { d'un même traitement }
$$

le nombre de degrés de liberté étant respectivement de 7 et 8 (SNENECOR, 1956). Nous avons enfin caractérisé chaque candidat par la moyenne des $\mathrm{F}$ obtenus pour les 4 caractéristiques analysées (saveur, tendreté, jutosité, texture).

TABI,EAU I

Résultats des essais préliminaires en vue du choix des dégustateurs.

\begin{tabular}{|c|c|c|c|c|c|}
\hline \multicolumn{3}{|c|}{ Hommes } & \multicolumn{3}{|c|}{ Femmes } \\
\hline No d'ordre & $\begin{array}{l}\text { Tests Trian. } \\
\text { bonnes répon. } \\
\text { en } \%\end{array}$ & $\begin{array}{l}\text { Dégustation } \\
\text { F moyelr }\end{array}$ & $x^{\circ}$ d'ordre & $\begin{array}{l}\text { Tests Trian. } \\
\text { bonnes répon. } \\
\text { en \% }\end{array}$ & $\begin{array}{l}\text { Dégustation } \\
\text { F moyen }\end{array}$ \\
\hline 1 & 80 & 3,73 & 11 & 60 & 6,33 \\
\hline $2^{*}$ & 93 & 3,15 & $12^{*}$ & 100 & 4,02 \\
\hline $3^{*}$ & 60 & $3,0_{t}^{\prime}$ & $13^{*}$ & 100 & 3,92 \\
\hline $4^{*}$ & 100 & 2,94 & $1: *$ & 70 & 3,31 \\
\hline 5 & 70 & 2,55 & 15 & 100 & 2,82 \\
\hline 6 & 100 & 2,34 & 16 & 100 & 2,68 \\
\hline 7 & 100 & 2,00 & 17 & 73 & 241 \\
\hline $8^{*}$ & 60 & 1,75 & $18 *$ & 80 & 2,31 \\
\hline 9 & 80 & 1,65 & 19 & 100 & 9,15 \\
\hline 10 & 47 & 1,49 & 20 & 100 & 1,71 \\
\hline non classé & 100 & $\begin{array}{l}\text { absent aux } \\
\text { dégustations }\end{array}$ & non classée & 90 & abandon \\
\hline
\end{tabular}

* Candidats définitivement choisis comme dégustateurs.

Le tableau 1 indique les résultats de nos tests de sélection. Les candidats ont été choisis d'après la valeur du F moyen. Mais pour garder le même jury tout au long des 8 semaines d'expérience, nous avons dû éliminer ceux qui ne pouvaient matériellement pas satisfaire à la condition d'assister à toutes les dégustations. Ainsi nous avons dû remplacer les candidats no $n^{0}$ et I i par les les $n^{\circ} 8$ et 18 . De plus, par suite d'une absence non prévisible au départ, le dégustateur no i 3 a été remplacé par le $n^{0}$ is pendant la cinquième semaine. 


\section{RÉSULTATS \\ I. - Variabilité et corrélations phénotypiques}

Le tableau 2 donne la moyenne sur io (m), l'écart-type (s) et le coefficient de variation (C. V. exprimé en p. IOO) pour chacune des caractéristiques étudiées.

T.ABLEAU 2

Moyenne (m), écart-type (s) et coefficient de variation en p. 100 (CV), par caractère organoleptique et par morceau.

\begin{tabular}{|c|c|c|c|c|c|c|c|c|c|}
\hline \multirow{2}{*}{ Caractère } & \multicolumn{3}{|c|}{ Blanc } & \multicolumn{3}{|c|}{ Cuisse } & \multicolumn{3}{|c|}{ Peaul } \\
\hline & $m$ & $s$ & $\mathrm{Cr}$ & $m$ & $s$ & $\mathrm{CV}$ & $m$ & $s$ & $\mathrm{CV}$ \\
\hline Qualité saveur............ & 7,05 & 0,11 & 5,8 & 7,35 & 0,37 & 5,0 & 7,06 & 0,61 & 8,6 \\
\hline Intensité goùt $\ldots \ldots \ldots \ldots$ & 5,55 & $0, \overline{5} \mathbf{4}$ & 9,7 & $6,2: 3$ & 0,58 & 9,3 & 6,33 & 0,71 & $11,: 2$ \\
\hline Tendrete $\ldots \ldots \ldots \ldots \ldots$ & 7,63 & 0,70 & 9,2 & $6,9^{\prime}$ & 0,88 & 12,7 & 6,13 & 1,23 & $\simeq 0,1$ \\
\hline Jutosité........ & 6,11 & 0,83 & $13,6 i$ & $7,2,4$ & 0,57 & 7,9 & 6,09 & $1,0:$ & 17,2 \\
\hline Texture $\ldots \ldots \ldots \ldots \ldots$ & 7,12 & 0,60 & 8,5 & 7,40 & 0,62 & 8,4 & 6,90 & 0,81 & 13,2 \\
\hline Moyenne arith. des 5 caract.. & 6,69 & 0,45 & 6,7 & $7,0^{\prime}$ & $0, \mathbf{1}^{\prime}$ & 6,3 & 6,50 & 0,78 & $1 \geq, 0$ \\
\hline Notes de $\operatorname{rang} \ldots \ldots \ldots$. & 5,00 & 0,38 & 7,$6 ;$ & 5,00 & 0,37 & 7,5 & 5,00 & $0,4^{\prime}$ & 8,8 \\
\hline
\end{tabular}

Les morceaux ont été comparés 2 à 2 en appliquant aux moyennes indiquées dans le tableau 2 le test $t$ de Student-Fischer : $t=d / s_{t}$ où $d$ exprime la différence entre 2 moyennes. L'écart type de la différence, $s_{d}$, a été calculé en tenant compte des corrélations entre morceaux, selon la formule:

$$
s_{l i}^{2}=s_{\bar{x}}^{2}+s_{\bar{y}}^{2}-2 s_{\bar{x}} s_{\bar{y}}
$$

Pour les corrélations significatives, on a pris les valeurs indiquées au tableau 4 . Pour les corrélations non significatives on a pris $r=0$.

TABLEAU 3

Valeur absolue des différences entre morceaux (d) et valeur du " $t$ " correspontant pour chaque caractire organoleptique.

\begin{tabular}{|c|c|c|c|c|c|c|}
\hline \multirow{2}{*}{ Caractère } & \multicolumn{2}{|c|}{ Blanc-Cuisse } & \multicolumn{2}{|c|}{ Cuisse-Peau } & \multicolumn{2}{|c|}{ Blanc-Peau } \\
\hline & $d$ & $t$ & $d$ & $t$ & $d$ & $t$ \\
\hline Qualité saveur & 0,30 & $5,2 * * *\left({ }^{1}\right)$ & 0,29 & $4,4^{* * *}$ & 0,01 & 0,13 N.S. \\
\hline Intensité grout $\ldots \ldots \ldots \ldots \ldots$ & 0,68 & $2,8 * *$ & 0,10 & 1,3 N.S. & 0,78 & $8,3^{* * *}$ \\
\hline Tendreté.......... & 0,69 & $5,8^{* * *}$ & 0,81 & $5,1 * * *$ & 1,50 & $10,0 * * *$ \\
\hline Jutosité . . & 1,13 & $12,8 * * *$ & 1,15 & $9,3 * * *$ & 0,0 & 0,14 N.S. \\
\hline Texture.... & 0,28 & $8,5^{* * *}$ & 0,50 & $5,3 * * *$ & 0,22 & $2,4^{*}$ \\
\hline Moyenne arith. des 5 caractères. & 0,35 & $6,2^{* * *}$ & $0,5^{\prime} t$ & $6,5 * * *$ & 0,19 & $2,0^{*}$ \\
\hline
\end{tabular}

(1) *** valeur de $t$ significative au seuil 0,1 p. 100 .

** valeur de $t$ significative au seuil 1 p. 100.

* valeur de $t$ significative au seuil 5 [3. 100.

N.S. Valeur de $t$ non significative. 
Le tableau 3 donne les différences et les valeurs de $t$ correspondantes. Toutes, sauf trois, sont significatives.

Le tablea1 4 donne les corrélations phénotypiques entre morceaux, pour un caractère organoleptique donné. Les valeurs trouvées sont légèrement positives ou non significatives.

TABIEAU 4

Corrélaticns phénotypiques entre morceaux pour un caractère organoleptique donns.

\begin{tabular}{|c|c|c|c|}
\hline Caractère & Blanc-Cuisse & Cuisse-Peau & Blanc-Peau \\
\hline 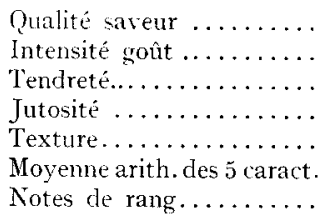 & $\begin{array}{l}0,15 \text { N.S. (1) } \\
0,21^{*} \\
0,17 \text { N.S. } \\
0,33^{* *} \\
0,25^{*} \\
0,39^{* * *} \\
0,11 \text { N.S. }\end{array}$ & $\begin{array}{l}0,28 * * \\
0,37 * * * \\
0,09 \text { N.S. } \\
0,11 \text { N.S. } \\
0,24^{*} \\
0,28^{* *} \\
0,21^{*}\end{array}$ & $\begin{array}{r}-0,02 \text { N.S. } \\
0,03 \text { N.S. } \\
-0,17 \text { N.S. } \\
0,11 \text { N.S. } \\
-0,15 \text { N.S. } \\
-0,07 \text { N.S. } \\
-0,10 \text { N.S. }\end{array}$ \\
\hline
\end{tabular}

(1) N.S. Valeur non significativement différente de zéro.

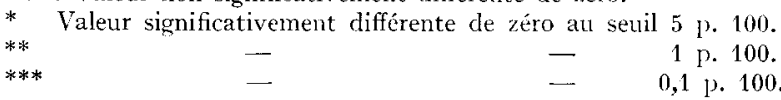

Le tableau 5 donne, pour chaque morceau, les corrélations phénotypiques entre caractères organoleptiques pris 2 à 2 . Les valeurs obtenues sont nettement plus grandes, une seule n'étant pas significative.

TABLEAU 5

Corrélations phénotypiques entre caractères organoleptiques pour un morceau donné.

\begin{tabular}{|c|c|c|c|}
\hline Caractères en corrélation & Blanc & Cuisse & Peau \\
\hline Saveur-Int. goût... & $0,45^{* * *}(1)$ & $0,61 * * *$ & $0,55^{* * *}$ \\
\hline Saveur-Tendreté ... & $0,53^{* * *}$ & $0,45 * * *$ & $0,68^{* * *}$ \\
\hline Saveur-Jutosité ........ & $0,80^{* * *}$ & $0,59 * * *$ & $0,68^{* * *}$ \\
\hline Saveur-Texture ......... & $0,60 * * *$ & $0,56 * * *$ & $0,75 * * *$ \\
\hline Int. goût-Tendreté ....... & 0,20 N.S. & $0,33 * * *$ & $0,48^{* * *}$ \\
\hline Int. goùt-Jutosité........ & $0,27^{* * *}$ & $0,35 * * *$ & $0,58 * * *$ \\
\hline Int. goût-Texture ........ & $0,22 *$ & $0,{ }^{\prime} 0^{* * *}$ & $0,60 * * *$ \\
\hline Tendreté-Jutosité ........ & $0,41 * * *$ & $0,35 * * *$ & $0,47 * * *$ \\
\hline Tendreté-Texture........ & $0,52 * * *$ & $0,60^{* * *}$ & $0,82 * * *$ \\
\hline Jutosité-Texture ........ & $0,47^{* * *}$ & $0,41^{* * *}$ & $0,56 * * *$ \\
\hline
\end{tabular}

(1) X. S. Valeur non significativement différente de zéro.
* Valeur signifiirativement différente de zéro au seuil 5 p. 100.
** - $\quad$ - $\quad 1$ p. 100.
*** - $\quad-\quad 0,1$ p. 100.

\section{2. - Variabilité d'origine génétique}

Elle a été appréciée en faisant une analyse de variance sur les go poulets pour chaque caractère étudié. La variabilité « entre coqs " et la variabilité " entre poulesintra coqs " ont été comparées à variabilité " entre pleins frères " à l'aide du test $\mathrm{F}$ 
de Fischer-Snedecor (SxEDECOR, I956). Le tableau 6 donne les valeurs de F obtenues.

TABLEAU 6

Analyse de variance entre les 90 poulets.

Valeurs obtenues pour F par caractire organoleptique et par morceau.

\begin{tabular}{|c|c|c|c|c|c|c|}
\hline \multirow[b]{2}{*}{ Caractère } & \multicolumn{2}{|c|}{ Blans: } & \multicolumn{2}{|c|}{ Cuisse } & \multicolumn{2}{|c|}{ Peati } \\
\hline & Entre cogs & $\begin{array}{l}\text { Entre poules- } \\
\text { intra cogs }\end{array}$ & Entre coqs & $\begin{array}{l}\text { Entre poules- } \\
\text { intra cops }\end{array}$ & Entre roqs & $\begin{array}{l}\text { Eutre youles- } \\
\text { intra coqs }\end{array}$ \\
\hline Qualité saveur & $0,2 \overline{2}$ & 1,51 & 0,9 ' & 1,25 & 0,75 & 0,85 \\
\hline Intensité goût..... & $0,8:$ & 1,31 & 0,68 & 0,65 & 1,40 & $1,6^{\prime} \dot{x}$ \\
\hline 'lendreté......... & $1,7 x^{\prime}$ & 0,91 & $2,54 *$ & 1.57 & 1,35 & l.íl \\
\hline Jutosité . . . . . . . . & 0,50 & 1,1'́ & 1,97 & 1,60 & 0,72 & $0,8:$ \\
\hline Texture .......... & $0, \overline{5} 5$ & 0,47 & 2,03 & $1,0: 3$ & 1,233 & $1.81 *$ \\
\hline $\begin{array}{l}\text { Moyenne arith. des } 5 \\
\text { caracteres ........ }\end{array}$ & 0,37 & 1,00 & $2,16 *$ & 0,93 & 1,23 & 1.11 \\
\hline Notes de rang ..... & 0,66 & 1,13 & $2,19^{*}$ & 1,35 & 1,90 & 1.22 \\
\hline
\end{tabular}

* Valeur de F significative au seuil 5 p. 100 .

L'organisation de l'expérience permettait le calcul de l'héritabilité des différents caractères étudiés et on était dans le cas simple où les subclasses sont égales. Le tableau 7 donne les résultats obtenus pour l'héritabilité moyenne :

$$
h^{2}=\frac{I}{2}\left(h_{\text {pèr }}^{2}+h_{\text {mère }}^{2}\right)
$$

ainsi que l'écart-type $s$ de cette valeur moyenne. Nous avons utilisé les techniques de calcul indiquées pat Graybili, et Robrrtsox (I957).

TABLEAU 7

Valcurs de l'héritabilite $\left(h^{2}\right)$ et de l'ecart-lype correspondant par caratire organoleptique ct pay morcentu.

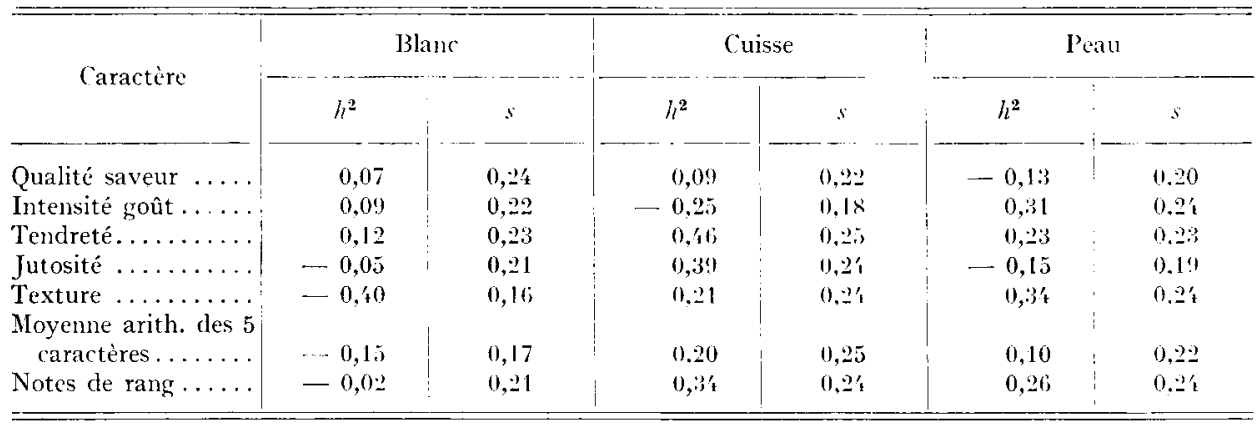

Pour obtenir l'intervalle de confiance correspondant au seuil de probabilité 5 p. IOo, il suffit de former la quantité $h^{2} \pm$ I, $96 \mathrm{~s}$. Pour toutes les valeurs positives du tableau 7 cet intervalle comprend zéro : aucune valeur trouvée pour l'héritabilité n'est donc significative. Ce résultat est une conséquence logique des chiffres consignés dans le tableau 6. 


\section{DISCUSSION}

\section{1. - Comparaison des iariabilités phénotypiques}

Les coefficients de variation indiqués dans le tableau 2 montrent que, d'une façon générale, les caractéristiques de la peau sont plus variables que celles du blanc et de la cuisse, principalement pour les caractères physiques de tendreté, de jutosité et de texture. Blanc et cuisse ont une variabilité analogue pour la saveur, l'intensité du goût et la texture. Pour la tendreté, la cuisse présente une variabilité plus grande que le blanc ; c'est le contraire pour la jutosité. Chez les dindons, Mardser et al. (I952) ont trouvé un résultat analogue. Pour nos poulets, on observe dans les 2 cas que l'écart-type le plus faible est lié à la plus forte moyenne : le jury semble faire moins de différence si la qualité qu'il juge a une moyenne élevée.

La saveur présente une variabilité assez faible par rapport aux autres caractères. Or les discussions qui avaient lieu après les séances de dégustation faisaient ressortir que les poulets étaient tous bons et peu différents les uns des autres. La remarque ci-dessus semble encore valable ici.

\section{2. - Valeur velative des morceaux}

Il n'est pas possible de comparer, pour un morceau donné, les moyennes entre caractères organoleptiques car les jugements, orientés par les feuilles de note à échelles descriptives, portent sur des caractéristiques peu comparables. Il est plus logique de comparer les morceaux pour un caractère donné (tableaux 2 et 3 ).

La saveur de la cuisse est significativement supérieure à celle du blanc et de la peau. La cuisse est également mieux notée que le blanc par le jury de FRONNING, Swanson et Benson (ig60) et celui de Kahlenberg, Pudeikewicz et Voss (Ig6i). Les résultats de MARGOLF et al. (I956) montrent qu'il $y$ a peu ou pas de différences entre cuisse et blanc alors que dans d'autres expériences les dégustateurs préfèrent la saveur du blanc: STEWAR'T, Hanson et LoWe (I943), Morrison et al. (I954), LEWrs et al. (I956). KAHLENBERG, PUDELKEWICZ et Voss pensent que ces différences sont dues à la méthode de cuisson qui n'est pas la même pour tous : les poulets de L EWIs et ceux de MORIsson ont été enveloppés d'une feuille d'aluminium avant d'être placés au four, les autres ont été cuit soit au four soit sur rôtissoire électrique. Il est probable que d'autres facteurs sont en cause tels que l'origine des poulets et peut-être un comportement différent des jurys de dégustation, question qui mériterait une étude approfondie.

Pour l'intensité du goût, cuisse et peau se valent et dominent le blanc. PIPPEN, CAMpbell et Streeter (I954) ont obtenu avec le jus de dialyse préparé à partir de la viande seule une saveur nettement plus forte qu'avec celui préparé à partir de la peau, mais ils n'ont pas trouvé de différence significative entre viande rouge et viande blanche. En faisant également une extraction à l'eau de différentes fractions de la carcasse, PETERSON (r957) trouve que les muscles de la cuisse donnent un jus à saveur très intense, la poitrine un jus moyennement intense et la peau un jus qui n'a qu'un faible goût de poulet. La forte intensité de gồt trouvée dans la peau de nos poulets a peut-être été acquise au cours de la cuisson par suite de transformations physicochimiques particulièrement importantes à ce niveau oul d'un mouvement centrifuge des substances responsables du goût. 
Les moyennes de tendreté font apparaître l'ordre décroissant suivant : blanc, cuisse, peau, les différences étant très hautement significatives. Ces résultats sont en accord avec ceux de nombreux auteurs : HANSOn, STEWART et LowE (I942), Morrison et al. (I954), BaKer (I957), Gilpix et al. (I960). I)e même, en étudiant individuellement i2 muscles du poulet, Strandine, Koonz et RamsbotTom (I949) observent que les plus tendres sont ceux de la poitrine (pectoralis superficialis et pectoralis profondus). KAHLExBERG, PUDELKEwicz et Voss (I96I) ont trouvé que la cuisse était plus tendre que le blanc, mais ils n'ont pris dans la cuisse qu'un muscle, le Semimembranosus, alors que la plupart des expérimentateurs utilisent un échantillon complexe comprenant au moins 3 muscles élémentaires.

I a jutosité de nos poulets se comporte comme la saveur : la cuisse est significativement plus juteuse que le blanc et la peau. Les animaux de STEWART, HAxsox et Lowe (I943) ainsi que ceux de Kahlenberg, Pudelkewicz et Voss (I96i) avaient eux aussi la cuisse plus juteuse que le blanc.

Les tableaux 2 et 3 montrent enfin que pour la texture et la moyenne des notes l'ordre préférentiel est le suivant : cuisse, blanc, peau.

Il est intéressant de remarquer que dans la plupart des cas, la cuisse est le morceau qui obtient les meilleures notes. Une enquête faite indépendamment des expériences de dégustation parmi nos 34 volontaires a montré que les deux tiers de ceux-ci avaient une préférence plus ou moins marquée pour la cuisse (la question posée était : "dans un poulet, préférez-vous le blanc ou la cuisse? ") : pour beaucoup de gens, au moins en France, la cuisse semble être le meilleur morceau du poulet.

\section{3. - Intérêt des relations entre morceaux et entre caractéristiques}

Les corrélations entre morceaux sont relativement peu importante et aucune valeur trouvée entre blanc et peau n'est significative (tableau 4). Il semble donc nécessaire de tenir compte à la fois des 3 morceaux pour contrôler la qualité d'ensemble du poulet.

Au contraire, pour un morceau donné, toutes les valeurs sont positives et significatives, à l'exception de la corrélation entre intensité du goût et tendreté du blanc (tableau 5). Pour les dindes, Marsnex et al. (1952) indiquent que la jutosité est en relation avec la tendreté et la saveur. Avec la viande de bœuf, KRopf et GRAF (I959) trouvent une corrélation significative entre tendreté et jutosité, une corrélation positive mais non significative entre saveur et tendreté, saveur et jutosité.

Il est difficile de savoir si les fortes valeurs que nous avons obtenues sont dues à une relation réelle entre les caractères étudiés ou bien si elles traduisent la tendance des dégustateurs à utiliser la même zone de notation sur les 5 échelles d'une même feuille. Quoi qu'il en soit, l'existence de telles cortélations suggère la possibilité de simplifier la feuille de note. A la limite, le seul jugement de la saveur permettrait d'avoir encore une bonne estimation de la qualité d'ensemble du morceau dégusté.

Le tableau 5 permet de constater qu'en moyenne la saveur présente les plus fortes corrélations avec les autres caractéristiques et l'intensité du goût les plus faibles, principalement en ce qui concerne le blanc. L'intensité du goût se présente donc comme un caractère relativement indépendant des caractères physiques (tendreté, jutosité, texture). Au contraire, la saveur leur est fortement liée, en particulier avec la jutosité du blanc. Tout se passe comme si la méthode utilisée pour juger la 
saveur aboutissait en fait à donner une note en relation avec tous les autres caractères, comme le ferait une note de "qualité globale». Cette observation paraît très générale : Pippen, Campbeli, et Strefter (I954) ainsi que Pilgrin et Schutz (I957) considèrent que la saveur est la résultante d'un complexe de sensations perçues au niveau de tous les récepteurs sensitifs de la bouche et du nez. Il est donc normal que la saveur soit en corrélation aussi bien avec les qualités gustatives proprement dites qu'avec les caractéristiques physiques perçues au moment de la mastication de l'échantillon.

\section{4. - Etude génétique des caractères organoleptiques}

L'étude de la variabilité génétique donne des résultats peu significatifs, malgré l'hétérogénéité actuelle de la souche utilisée. En effet, la variabilité " entre pleinfrères " est grande par rapport à la variabilité "entre coqs " et " entre poules intra-coqs". Le calcul de $\mathrm{F}$ (tableau 6 ) ne donne des valeurs significatives que pour la tendreté et la qualité globale de la cuisse (entre coqs) et la texture de la peau (entre mères). En conséquence, le calcul de l'héritabilité donne des valeurs faibles et non significatives (tableau 7 ).

Ces résultats concordent avec ceux de la littérature : il est difficile de mettre des différences en évidence pour les caractères organoleptiques entre groupes de poulets d'origine génétique différente. Ainsi dans une même souchede New-Hampshire Paul, Sorenson et Abplanat. (r959) ont trouvé que la tendreté présentait une variabilité aussi grande entre demi-frères, fils d'une même poule, qu'entre individus non parents. Entre poulets de race New-Hamsphire et 7 croisements, tous à base de New-Hampshire, Morrison et al. (1954) n'ont trouvé de différences significatives ni pour la saveur ni pour la tendreté. Mème entre races, Lowe (I948) note que les différences de saveur doivent être trop faibles pour être décelables par la méthode habituelle des tests de dégustation. C'est ce qu'on effectivement observé pour la saveur HAxsox et al. (I959) et pour la tendreté Gir.PIN et al. (I960) qui étudiaient pourtant des Plymouth-Rock barrés et un croisement New-Hampshire $\times$ Cornish. Au contraire, comparant les races Sussex, Rhode-Island rouge, Leghorn blanche, Polonaise à pattes vertes et leurs croisements, CHRzAszcz (I957) observe qu'au point de vue goût de la viande, les Sussex, les Sussex $\times$ Rhode et les Sussex $\times$ Polonaise sont meilleurs que les autres groupes.

Peut-on expliquer ces résultats souvent négatifs? En ce qui nous concerne, le faible nombre de poulets dégustés pouvait conduire à une erreur d'échantillonnage très importante. Il était peut-être présomptueux d'étudierla variabilité génétique d'une souche, pour des caractères aussi complexes que les qualités organoleptiques, avec seulement go poulets. Mais la méthode de dégustation directe ne permet pas d'analyser un grand nombre de poulets et ceci pose le problème de la recherche d'autres systèmes de mesure.

D'autre part, les résultats de 1'analyse de variance (tableau 6), laissent penser que le milieu joue un plus grand rôle que le génotype. Les poulets avaient déjà subi toutes les influences de l'environnement jusqu'à leur abattage. De plus, malgré le soin pris pour uniformiser les conditions de préparation des animaux, il est possible que de faibles variations au cours de l'abattage, de la conservation ou de la cuisson puissent suffire à masquer les différences d'origine génétique. 
Enfin, on peut se poser la question de la valeur de la méthode de dégustation pour de telles expériences. Notre jury a. fait des différences entre potulets, puisque les coefficients de variation ne sont pas négligeables (tableau 2). Mais l'expérience a duré 8 semaines et il est difficile do prouver que les réactions des juges ont été les mêmes au début qu'à la fin ou bien qu'elles ont évoluées avec le déroulement de l'expérience. D'autre part, la précision et la fidélité des jugements sont sujettes à caution car les valeurs moyennes de $\mathrm{F}$, qui caractérisent chaque dégustateur, sont assez faibles (tableau I). OVERMAx et Li (I948) ont observé un F variant de 5, I4 à 60,78 en faisant déguster Io fois à Io personnes (une fois par jour pendant ro jours) 5 séries de gâteaux confectionnés selon la même recette mais avec des matières grasses très différentes. Les conditions de cette expérience étaient très éloignées des nôtres mais la comparaison des résultats montre que la dégustation du poulet est chose peu précise, d'où les conclusions à priori pau significatives d'une analyse de variance.

La méthode des tests de dégustation reste cependant très utilisée et il est difficile de l'abandonner car elle mesure directement ce que pense et ce que désire le consonlmateur, but final de toute recherche agronomique. Il faut plutôt chercher à améliorer sa précision et multiplier les expériences analogues à celle-ci. On pourra alors discuter sur des données plus nombreuses et, peut-être, promouvoir des méthodes d'amélioration de la qualité organoleptique des souches de poulet.

\section{REMERCIEMENTS}

L'auteur tient particulièrement à remercier le personnal du Domaine du Magneraud qui a bien voulu participer aux épr unves éliminatoires, puis aux différentes séances de dégustation, et sans lequel cette expérience n'aurait pu être entreprise.

Rę̧u en septembre 1961.

\section{SUMMARY}

OBSERVATIONS ON ORGANOLEPTIC CHARACTERISTICS OF CHICKENS FRO II THE SAME STRAIN.

Palatability tests were performed on go twelve-weeks-and-three-days old roasted cockerels from the same strain. 8 judges ( 4 men and 4 women) were salected among 34 volunteers on their ability to detect chicken flavor in a diluted solution and their consistency during 2 training sessions. Results of these preliminary selection tests are s'mm tm triz:i in table I. During the experiment, 4 birds, were tested in each session. Skin taken on the back, breast and thigh meat were noted for havor quality, taste intensity, tendemess, juiciness and texture, by using a score card with descriptive scales (fig. I). The 4 chickens were als') ranked for over-all quality of skin, breast and thigh. Our results are given in tables 2 to 7 .

Table 2 gives means, standard deviations and variation coefficients. Phenotypic variability is greater for skin than for breast or thigh meat, lower for flavor than other characteristics.

Table 3 gives the significance of differences between breast and thigh, thigh and skin, skin and breast for each characteristic. The t-test was used. The thigh appears to be the best part of the chicken.

Table 4 gives phenotypic correlations between parts of the chicken. They are moderately posi1ive or not significant.

Table 5 gives phenotypic correlations between organoleptic characteristics, intra-parts of the chicken. Most of them are highly significant.

Table 6 gives the F-values of an analysis of variance : between sires/between full-brothers and between dams-intra sires/between full-brothers. Only thigh tenderness (between sires), skin texture (between dams) and over-all quality of thigh (between sires) lead to significant figures. 
Table 7 gives heritability values of each characteristic and their standard deviations. All are low and not significant.

The last two tables fail to show an obvious genetic variability between the 30 families studied. Several reasons are discussed to explain this result.

\section{RÉFÉRENCES BIBLIOGRAPHIQUES}

BAKER R. C., 1957. Determining the keeping quality of ice-packed and dry-packed poultry. Ponll. Sci., 36, 859-864.

Baker R. C., Naylor H. B., Pfund M. C., Einset E., Staempfli W., i956. Keeping quality of readyto-cook and dressed poultry. Poult. Sci., 35, 398-406.

Chamberlain F. W., i943. Atlas of avian anatomy. Michigan Agr. Exp. Sta., Mem. bull., 5, i68-I7ı.

CHRzaszcz 'T., I957. Comparison of the quality of meat of 4 breeds of chickens recognized in Poland and some of their crosses. Rocz. Nauk. rol. (B), ri1, 445-468. Résumé dans : World's Ponlt. Sci. J., 14, 248.

FISHER R. A., YATES F., I948. Statistical tables for biological, agricultural and medical research. Table XX p. 66. 3 rd. Ed., Oliver and boyd, London.

DE Fremery D., Pool. H. F., I960. Biochemistry of chicken muscle as related to rigor mortis and tenderization. Food Res., $25,73-87$.

Fronisg G. W., Swanson M. H., Benson H. X., rg6o. Moisture levels in frozen poultry as related to thawing losses, cooking losses, and palatability. Poull. Sci., 39, 373-377.

Gilpin G. L., Harkin A. M., Reostrom R. A., Dawson E. H., rg6o. Quality and yield of modern and old-type chickens. Poult. Sci., 39, 924-930.

Girardot N. F., Peryam D. R., Shapiro R., 1952. Selection of sensory testing panels. Food Technol, 6, I $40-143$.

GraybiLl F. A., Robertson W. H., 1957. Calculating confidence intervals for genetic heritability. Poull. Sci., 36, 26I-265.

Hanson H. L., Campbell A. A., Kraft A. A., Gilpix G. L., Hakkin A. M., 1959. The fiavor of modern and old-type chickens. Poult. Sci., 38, 1071-1078.

Hanson H. I., Stewart G. F., Lowe B., r942. Palatability and histological changes occuring in NewYork dressed broilers held at $1.7^{\circ} \mathrm{C}$. Food Res., 7, r48-160.

Kahlenberg O. J., Pudelkewicz C., Voss L. A., i96r. Factors affecting poultry flavor. The effect of semi-synthetic diet and pratical type rations on the palatability of broilers. Poult. Sci., 40, 634-638.

KROPF D. H., GRAF R. L., 1959. Interrelationships of subjective, chemical and sensory evaluations of beef quality. Food technol., 13, 492-495.

Lewis R. W., SAnford P. E., Ericson A. T., IIArrison D. L., ClegG R. E., ig56. Flavor and aroma of birds fed purified and standard diets. Poult. Sci., 35, 25I-253.

Lowe B., 1948. Factors affecting the flavor of poultry with emphasis on histological post-mortem changes Advances in jood Research, 1, 203-256.

Margolf P. H., Callenbach E. W., Miller R. C., Nicholas J. E., Perry J. S., Cone J. F., Murphy J. F., DodDs M. L., BENnet G., I956. The effect of type of scald and wrap on the market quality of frozen poultry. Poult. Sci., 35, 37-46.

Mardsen S. J., Alexander L. M., Schopmeyer G. F., Lamb J. C., I952. Varicty as a factor in fleshing, fatness and edible quality of turkeys. Ponlt. Sci., 31, 433-444.

Morrison M. A., Sauter E. A., Mclarex B. A., Stadelman W. J., 1954. Some factors affecting yield and acceptability of chicken broilers. Poult. Sci., 33, I I 22-I I 25 .

Overman A., LI J. C. R., I 948 . Dependability of food judges as indicated by an analysis of scores of a food tasting panel. Food Res., 13, $44 \mathrm{I}-4+40$.

Paul P. C., Sorenson C. I., AbPlanalp II., 1959. Variability in terderness of chicken. Food Res., 24, $205-209$.

PETERSON D. W., 1957. The source of chicken flavor. In Chemistry of natural food flanors, 167-173. U. S. Army Quartermaster.

PiLGRIM F. J., SchUTZ H. G., I957. Measurement of the qualitative and quantitative attributes of flavor In Chemistry of natural food flavors, $47-55 . \mathrm{U}$. S. Army Quartermaster.

Pippen E. L., Campbell A. A., Streeter I. V., i954. Flavor studies : origin of chicken flavor. J. Agric. Food chem., 2, 364-367.

Plank R. P., I948. A rational method for grading food quality. Food Technol., 2, $241-25$ I.

RAMSBotTom J. M., I947. Cité par PLANK R. P., I948. A rational method for grading food quality. Food Technol., 2, 24r-25I.

Rheinhold J., r940. Cité par PLANk R. P., I948. A rational method for grading food quality. Food Technol., 2, 24I-25i.

SNEDECOR G. W., 1956. Statistical methods, 237-29o. 5th Edition, The Iowa State College Press, Ames.

STEwart G. F., HANSON H. L., LowE B., I 943 . Palatability studies on poultry. A Comparison of 3 methods for handling poultry prior to evisceration. Food Res., 8, 202-211.

Strandine E. J., Koonz C. H., Ramsbottom J. M., I949. A study of variation in muscles of beef and chickens. J. Anim. Sci., 8, 483-494. 